\title{
Le conte de la grenouille ébouillantée
}

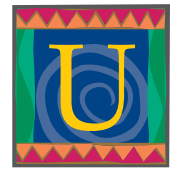

ne légende urbaine affirme que si l'on plonge une grenouille dans l'eau bouillante, elle bondira instantanément pour fuir, mais que si l'eau est agréablement tiède au début et qu'on la fait chauffer graduellement, la grenouille y demeurera jusqu'à ce qu'elle soit ébouillantée et meure. La grenouille serait en effet incapable de détecter à temps l'augmentation graduelle de la température.

On utilise souvent cet exemple en économie, en affaires et en marketing pour signaler que le changement doit être graduel pour réussir. L'exemple illustre aussi un aspect de la psychologie humaine : nous avons tendance à accepter les choses qui s'implantent lentement mais régulièrement, même lorsqu'elles en viennent à contrôler notre vie. Nous finissons toutefois par nous réveiller un jour et nous retrouver dans l'eau bouillante. Telle est la nature de l'excès.

Dans ce numéro des Fêtes, nous jetons un coup d'œil sur divers excès dans le domaine médical. Marcia Angell ${ }^{1}$ parle des habitudes excessives de l'industrie pharmaceutique (page 1451); Mary Seeman ${ }^{2}$ (page 1477) présente une rétrospective historique des excès de la psychiatrie au cours des années 1960; Farrah Mateen, ${ }^{3}$ étudiante en médecine, jette un coup d'œil sur la poursuite excessive du succès chez les nouveaux arrivants en faculté de médecine, mais aussi sur la représentation insuffisante de la société au sein de ce groupe (page 1466); tandis qu'un éducateur distingué, Eugene Stead ${ }^{4}$, pose des questions fondamentales sur le monopole historique des facultés de médecine (page 1465).

Nos contributeurs jettent un coup d'œil amusant ou sérieux sur d'autres formes d'excès. Comprenant comment les annonces des sociétés pharmaceutiques captent l'attention des consommateurs et orientent notre comportement, Steven Shumak et Donald Redelmeier ${ }^{5}$ présentent une leçon sur la façon de «lire» les annonces sur les médicaments afin d'être mieux en mesure de prendre une décision éclairée avant d'acheter (ou de prescrire) un nouveau produit qui promet de nous aider à réduire notre tour de taille, notre taux de cholestérol, notre tension artérielle, à at- ténuer notre douleur, nos pensées ou nos émotions (page 1455). Pour Steven Lewis', si nos attentes à l'égard de ce que les produits pharmaceutiques peuvent faire pour nous sont excessives, ce que nous attendons de l'ensemble des soins de santé l'est aussi. En fin de compte, il nous rappelle sans détour que nous ne sommes toujours pas en bien meilleure santé même si la facture des soins de santé gonfle de milliards de dollars par année (page 1449).
Tous ces excès sont-ils un signe de prospérité et de réussite matérielle? Nous mangeons non parce que nous avons faim, mais parce que la nourriture est disponible et abordable. Nous achetons non par besoin, mais par désir de posséder. Pourquoi nous contenter du nez que la nature nous a donné s'il est possible de le modifier chirurgicalement plus d'une fois? Pourquoi avoir seulement une automobile lorsque nous pouvons en posséder trois? Pourquoi vivre en appartement lorsqu'il est possible d'être propriétaire? Comme le dit si bien un ami, celui qui meurt avec le plus de jouets gagne.

Nous déterminons de façon sélective ce qui constitue ou non un excès. Il y a d'un côté l'obésité et de l'autre, l'anorexie, deux industries en croissance. Notre courbe gaussienne en cloche s'aplatit et le domaine extra-normal s'élargit. Certains, comme Joan Johnston ${ }^{7}$, soutiendraient que notre réaction à l'épidémie d'obésité est en soi excessive (page 1459).

Nous en sommes à la période de l'année où nous cédons à la folie du magasinage pour acheter des dindes de 25 livres, des boîtes de chocolat de 10 livres, une caisse de 24 ou même trois, et des tonnes de cadeaux de toutes sortes. Cette manie qui précède le temps des Fêtes est suivie d'une période de dépression financière et affective - qu'il est peut-être possible d'atténuer dans le dernier cas. Nous vivons dans la surabondance tandis que d'autres manquent constamment d'aliments, de vêtements et d'eau. Or ce n'est pas que nous manquons de ressources pour tous, mais plutôt que la répartition de ces ressources est inégale. Nous devrions peut-être envisager d'équilibrer la balance. Nous pourrions acheter une chèvre pour une famille affamée d'un village de la Sierra Leone au lieu d'acheter une autre cravate en soie pour papa. De tels efforts aident à rétablir l'équilibre, à élargir la courbe gaussienne. Sinon, le temps des Fêtes deviendra rapidement une maladie classifiable et nous devrons prendre des Noëlones pour y survivre. - $7 A M C$

\section{Références}

1. Angell M. Excess in the pharmaceutical industry. FAMC 2004;171(12):1451-3.

2. Seeman MV. Therapy in the gray zone: psychiatry recalled. $7 A M C 2004 ; 171$ (12):1477-9.

3. Mateen F. Darwinian pursuits with interruptions by Huxley: a brief pontification on medical education. $7 A M C$ 2004;171(12):1466-8.

4. Stead EA. Wisdom from a medical elder. 7AMC 2004;171(12):1465-6.

5. Shumak SL, Redelmeier DA. How to read clinical journals: XII. How you too can profit from pharmaceutical advertisements. FAMC 2004;171(12):1455-6.

6. Lewis S. Confronting the Christmas of our health care discontent. $7 A M C$ 2004;171(12):1449-50.

7. Johnson JM. Eating disorders and childhood obesity: Who are the real gluttons? 7AMC 2004;171(12):1459-60. 